\title{
A Flexible Blue Light-Emitting Diode Based on ZnO Nanowire/Polyaniline Heterojunctions
}

\author{
Y. Y. Liu, ${ }^{1}$ X. Y. Wang, ${ }^{1}$ Y. Cao, ${ }^{2}$ X. D. Chen, ${ }^{1}$ S. F. Xie, ${ }^{1}$ X. J. Zheng, ${ }^{1}$ and H. D. Zeng ${ }^{2}$ \\ ${ }^{1}$ School of Materials Science and Engineering, University of Shanghai for Science and Technology, Shanghai 20093, China \\ ${ }^{2}$ School of Materials Science and Engineering, East China University of Science and Technology, Shanghai 200235, China \\ Correspondence should be addressed to X. Y. Wang; xianyingwang@usst.edu.cn and H. D. Zeng; huidanzeng@ecust.edu.cn
}

Received 14 June 2013; Accepted 5 September 2013

Academic Editor: Wen Lei

Copyright (c) 2013 Y. Y. Liu et al. This is an open access article distributed under the Creative Commons Attribution License, which permits unrestricted use, distribution, and reproduction in any medium, provided the original work is properly cited.

\begin{abstract}
An organic/inorganic light-emitting diode (LED) consisting of n-type vertically aligned $\mathrm{ZnO}$ nanowires (NWs) and p-type proton acid doped polyaniline (PANi) is reported. The device was fabricated on flexible indium-tin-oxide (ITO) coated polyethylene terephthalate (PET) substrate. A broad blue light emission band ranging from $390 \mathrm{~nm}$ to $450 \mathrm{~nm}$ was observed in the electroluminescence (EL) spectra of the device, which was related to the interface recombination of electrons in the conduction band of ZnO NWs and holes in the polaron level of PANi. The turn-on voltage of the device is $\sim 3.5 \mathrm{~V}$, lower than most of ZnO NWs based LED devices. In combination with the easy fabrication, flexibility, low power consumption, and mechanical robustness, this novel device is very promising in the application of blue LEDs.
\end{abstract}

\section{Introduction}

$\mathrm{ZnO}$, especially in its nanostructured form, has been considered to be attractive for applications in ultraviolet (UV) lasers [1], LEDs [2-4], and solar cells [5] because of the wide band gap and high exciton binding energy. However, it remains critical and challenging to fabricate high quality $\mathrm{ZnO}$ with stable p-type conductivity because of the intrinsically $n$-type nature. The difficulty in doping $\mathrm{ZnO}$ to $\mathrm{p}$-type polarity has led the researchers to seek to create heterojunctions with other $\mathrm{p}$ type semiconductors to enable the use of $\mathrm{ZnO}$ in all kinds of electronic devices. Many efforts have been made to grow ntype $\mathrm{ZnO}$ on p-type substrates, but usually the quality of such heterojunctions is limited by the lattice mismatch. P-type conducting polymer has many advantages including easy fabrication, flexibility, and tunable carrier densities. Various LEDs based on ZnO NWs/p-type polymer heterostructures have been reported in the literature $[3,4]$; however, blue LED is still quite limited [6].

In this work, a blue LED device consisting of vertically aligned ZnO NWs and p-type conducting PANi heterojunction was fabricated. PANi was selected as the hole conducting layer because of its facile synthetic process, good environmental stability, easy conductivity control, and cheap production in large quantities. The structure of the LED device was schematically illustrated in Figure 1. Vertically aligned $\mathrm{ZnO}$ NWs are used as the light emission units, and proton doped PANi is used as the hole-transporting layer. Au film serves as the top electrode, and ITO is the bottom electrode. The function of polymethyl methacrylate (PMMA) is to form a smooth surface for PANi coating. A broad blue light emission band ranging from $390 \mathrm{~nm}$ to $450 \mathrm{~nm}$ was observed from the EL spectra of the $\mathrm{ZnO}$ NWs/PANi heterojunction LED. The origins of the blue emission were also discussed in this paper.

\section{Experimental}

For the LED device fabrication, $\mathrm{ZnO} \mathrm{NW}$ arrays were grown on ITO coated PET substrate using a low temperature hydrothermal method. Equimolar $(0.05 \mathrm{M})$ of hydrated zinc nitrate $\left(\mathrm{Zn}\left(\mathrm{NO}_{3}\right)_{2 \mathrm{~A}} \cdot 6 \mathrm{H}_{2} \mathrm{O}\right)$ and hexamethylenetetramine (HMT) was used as the precursor. The reaction was carried out at $90^{\circ} \mathrm{C}$ for 6 hours. After growth the as-grown NW forest was infiltrated with 5\% (weight percent) PMMA solution (in toluene) to provide a smooth film for subsequent PANi coating. Then oxygen plasma etching was applied to remove excessive PMMA and expose the NW tips for junction formation with PANi. $\mathrm{HCl}$ doped PANi dissolved in dimethylformamide (DMF) was spin coated on top of $\mathrm{ZnO} \mathrm{NW}$ tips. The device was then baked at $60^{\circ} \mathrm{C}$ for 10 hours to completely remove 
the organic solvents. Finally Au top electrode with the thickness of $150 \mathrm{~nm}$ was deposited using e-beam evaporation with the deposition rate of $0.1 \mathrm{~nm} / \mathrm{s}$.

The morphologies and microstructures of the as-synthesized $\mathrm{ZnO} \mathrm{NW}$ arrays were examined using scanning electron microscope (SEM, FEI Quanta FEG), transmission electron microscope (TEM, FEI G30), and X-ray diffraction pattern (XRD, Bruker D8 Advance). Electrical properties of the devices were examined using semiconducting characterization system (SCS, Keithley 4200) equipped with a probe station (Cascade M 150). The photoluminescence (PL) and EL spectra were detected using a FS 920 type spectrometer (Edinburgh).

\section{Results and Discussions}

From the SEM image of the as-grown $\mathrm{ZnO} N W$ arrays (Figure 2(a)), it can be seen that ZnO NWs are uniformly distributed on the PET substrate and primarily vertically aligned. The diameter of $\mathrm{ZnO} N W$ s ranges from $80 \mathrm{~nm}$ to $130 \mathrm{~nm}$, and the average height is around $3 \mu \mathrm{m}$. The TEM image of a randomly picked individual NW is shown in Figure 2(b). Clearly, the NW has uniform diameter of $110 \mathrm{~nm}$ throughout the entire length. The selected area diffraction pattern shown in the inset of Figure 2(b) demonstrates that the NWs are single crystalline with preferred $\langle 0001\rangle$ growth direction. Strong (0002) diffraction peak centered at $34.4^{\circ}$ can be observed from XRD pattern of the NW arrays (Figure 2(c)), suggesting that the NWs are highly ordered along $c$-axis with the lattice spacing of $0.52 \mathrm{~nm}$. No other impurity peaks or ITO related peaks are observed. The PL spectra of the as-grown $\mathrm{ZnO} N W$ arrays are presented in Figure 2(d). The strong ultraviolet emission peak centered at $383 \mathrm{~nm}$ is the intrinsic luminesce of $\mathrm{ZnO}$ NWs. The defect related visible emission at around $500 \mathrm{~nm}$ is much weaker than the UV peak, indicating that $\mathrm{ZnO}$ NWs are of high degree of crystalline quality with few defects.

Figure 3(a) shows the top-view SEM image of the surface of the device after PANi deposition. The bright spots represent the tips of $\mathrm{ZnO} \mathrm{NWs}$, and the dark area is the space between NWs solidly filled with PMMA. Obviously the NWs are well separated, demonstrating that no agglomerations occurred during the polymer infiltration process. The density of the NW tips can be estimated to be $14 \mathrm{NW} / \mu \mathrm{m}^{2}$. Figure 3(b) shows the current-voltage (IV) characteristics of the $\mathrm{ZnO}$ NWs/PANi heterostructure LED device. The IV curve clearly shows nonlinear increase of the current under the forward bias, which indicates the formation of $p-n$ junction between $\mathrm{ZnO}$ NWs and PANi. The turn-on voltage of the device is around 1.3 V, as shown in Figure 3(b).

The EL spectra of the ZnO NWs/PANi heterojunction LED device were measured under different forward biasing voltages $(2 \mathrm{~V}, 3.5 \mathrm{~V}, 5 \mathrm{~V}$, and $6.5 \mathrm{~V})$. As is shown in Figure 4(a), the light emission is too low to be detected when the forward bias is below $2 \mathrm{~V}$. However when the bias voltage is larger than $3.5 \mathrm{~V}$, a broad blue light emission band spreading through $390 \mathrm{~nm}$ to $450 \mathrm{~nm}$ can be observed. Obviously, this band is neither related with the near band edge nor to the deep level

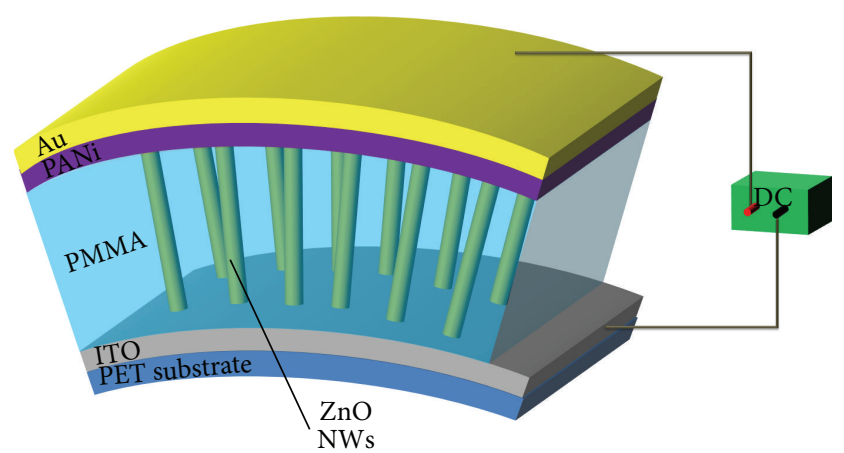

FIgURE 1: Schematic diagram of the flexible LED device. The basic structure of the device is $\mathrm{Au} / \mathrm{PANi} / \mathrm{ZnO} \mathrm{NWs}$ in PMMA matrix/ITO/PET substrate.

emission of ZnO NWs. Blue LEDs based on ZnO NWs/p$\mathrm{GaN}$ [2] and $\mathrm{ZnO} \mathrm{NWs} / \mathrm{PFO}[7]$ heterojunctions have been reported before; however, the emission is often dominated by the p-type conducting layer instead of $\mathrm{ZnO}$ NWs. To understand the origins of the blue light emission of $\mathrm{ZnO}$ NWs/PANi device, the energy band diagrams of all the layers were schematically illustrated in Figure 4(b). Doping caused a self-localized polaron band in PANi, existing as a defect band in the bandgap $(3.4 \mathrm{eV})$ between the valence band and the conduction band. It is half-filled and has a significant band width $(\sim 1.1 \mathrm{eV})$ [8-10]. When a forward bias is applied, electrons in the conduction band of the $\mathrm{ZnO}$ NWs will be driven to move towards the PANi. Also the holes in the polaron band of PANi will move towards $\mathrm{ZnO}$ because of the relatively low energy barrier. The electrons and holes recombine at the interface of the $p-n$ junction and produce a photon. The emission wavelength in the EL spectra must be corresponding with the bandgap between the conduction band of $\mathrm{ZnO}$ and the polaron band of PANi, although the exact position of the polaron band was not found in the literaturs.

Several other features can also be found from the EL spectra. First, the turn-on voltage of $\mathrm{ZnO} N W s / P A N i$ heterostructure is substantially lower compared with other $\mathrm{ZnO} \mathrm{NWs}$ based LED devices. For example, the turn-on voltage is $10 \mathrm{~V}$ for $\mathrm{ZnO} \mathrm{NWs} / \mathrm{p}$-type $\mathrm{GaN}$ film hybrid junction [2] and $6 \mathrm{~V}$ for $\mathrm{ZnO} \mathrm{NWs} / \mathrm{p}-\mathrm{Si}$ junction [11]. The small turn-on voltage can be attributed to the low carrier injection barriers (Figure 4(b)). Second, the blue emission peak is increasingly broadened, and the intensity is enhanced with the increase of the bias voltage. The broad blue light-emitting range suggests that the emission behavior is complicated and must be related to the polaron energy band of PANi. With the increase of the forward biasing voltage, more electrons in the conduction band of $\mathrm{ZnO}$ are driven to move towards the PANi, and more holes in the valence band of PANi will move towards $\mathrm{ZnO}$. Thus, a larger number of protons can be produced by the recombination of the carriers. Also, peak splitting in the blue region can be observed, which is not well understood. However, it is possible to be caused by the diffraction when the light transmits through hexagonal $\mathrm{ZnO}$ NWs. Third, the defect related visible light emission band is broadened, and the peak center is red shifted compared with that in the PL 


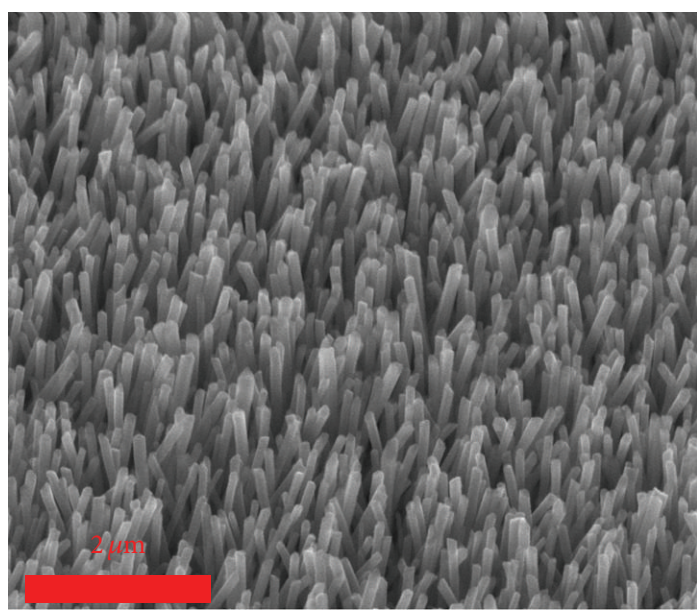

(a)

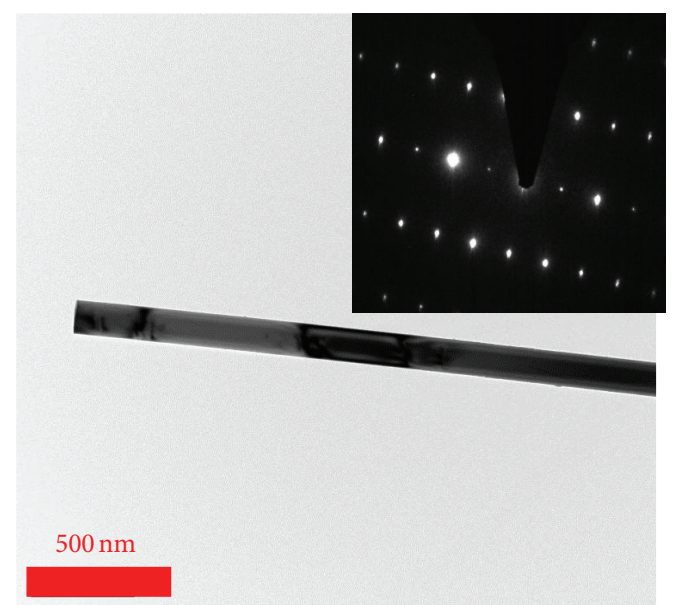

(b)

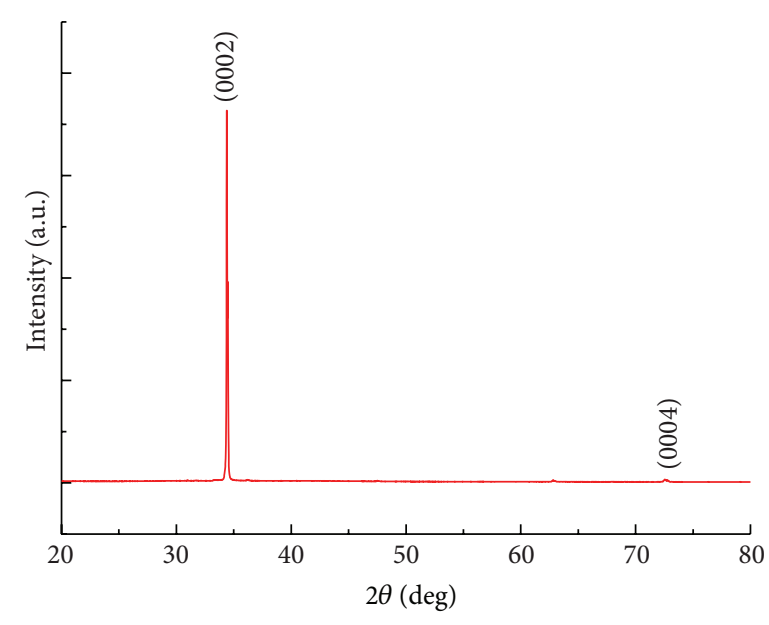

(c)

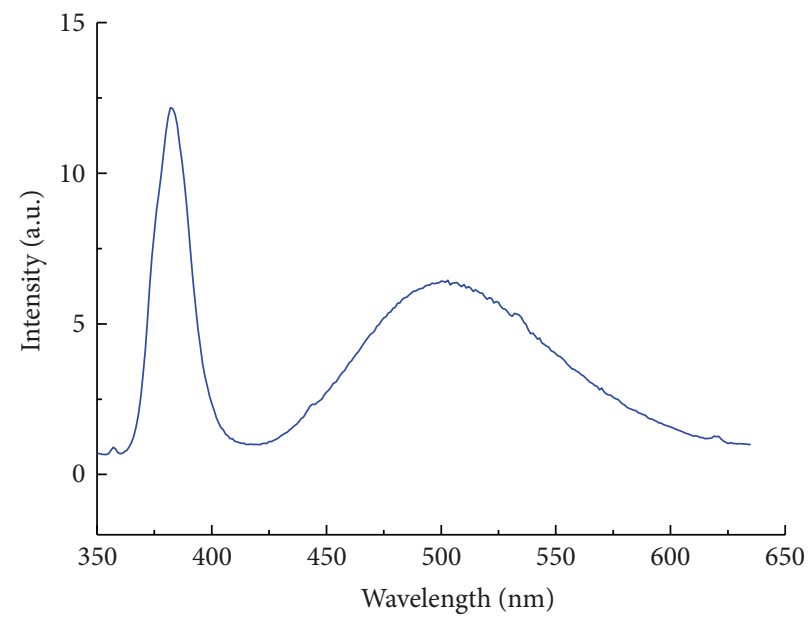

(d)

FIGURE 2: Morphology and structural characterizations of ZnO NWs. (a) SEM image. (b) TEM image. Inset: SAED pattern. (c) XRD curve. (d) PL spectra.

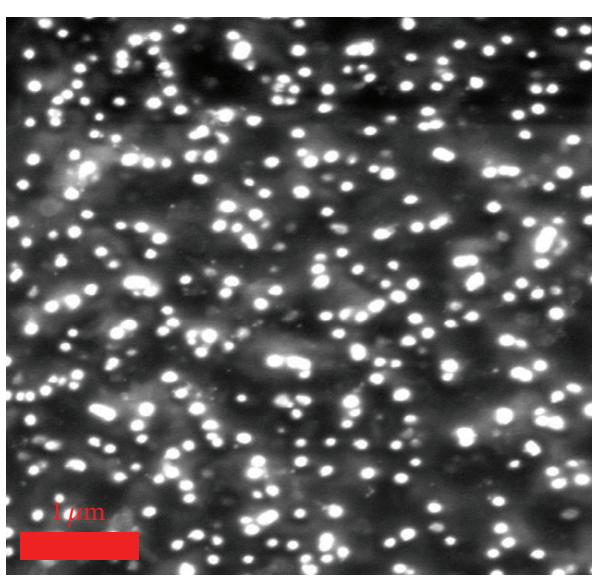

(a)

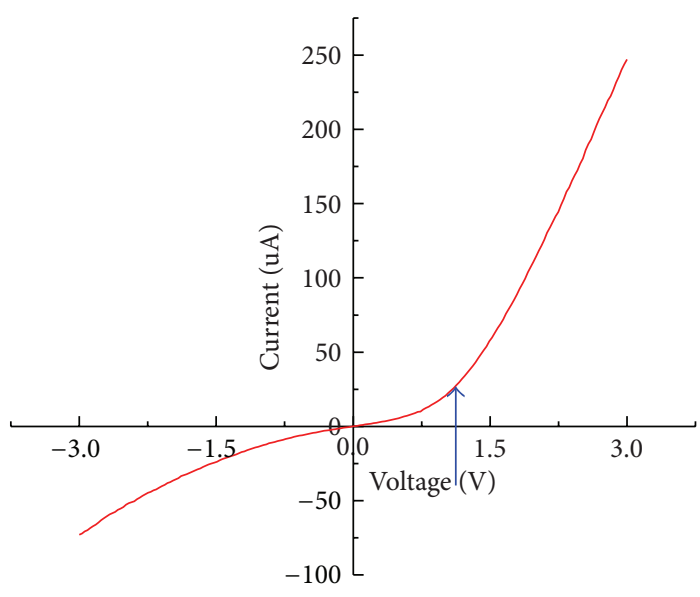

(b)

Figure 3: (a) Top view SEM image of the ZnO NWs/PMMA hybrids after PMMA infiltration. (b) IV curve of the ZnO NWs/PANi heterojunction blue LED device. 


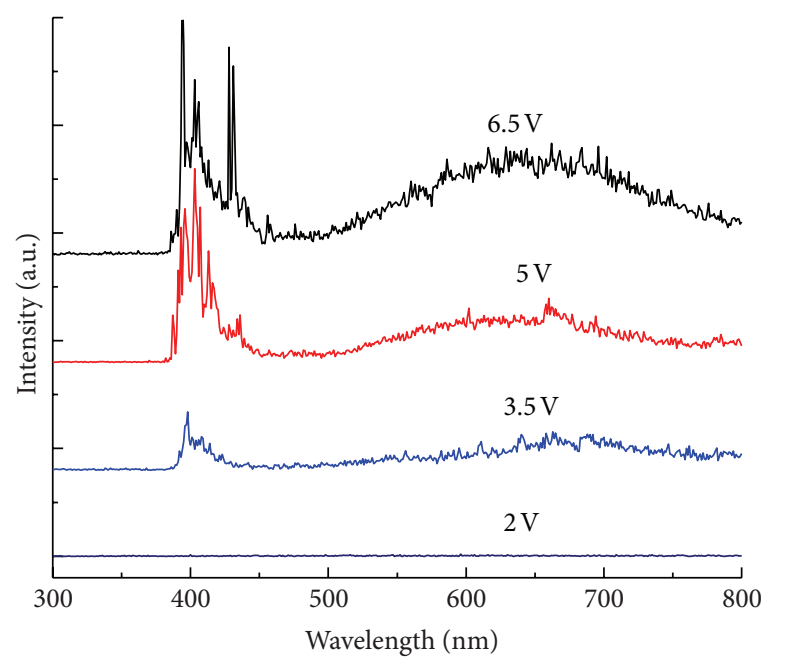

(a)

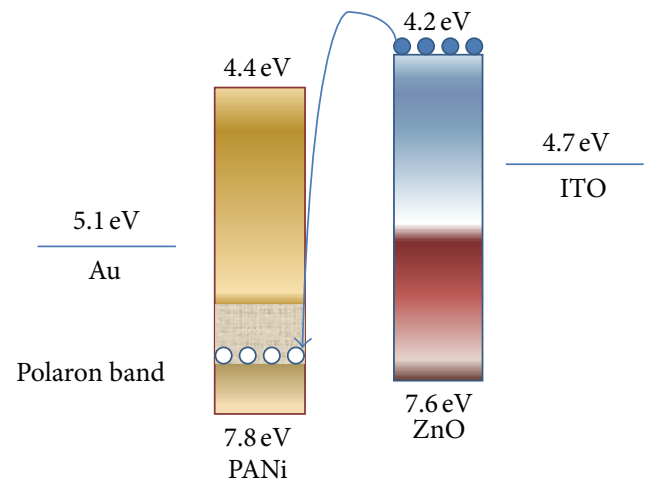

(b)

Figure 4: (a) EL spectrum of the ZnO NWs/PANi device measured under various biasing voltages. (b) Energy band diagrams of Au, PANi, ZnO NWs, and ITO layers.

spectra. This may be caused by the redistribution of the deep level defects in the $\mathrm{ZnO}$ NWs during the device fabrication process.

\section{Conclusions}

In this paper, an inorganic/organic heterostructure LED device based on vertically aligned $\mathrm{ZnO} \mathrm{NW}$ arrays and PANi conducting polymer was reported. A blue light emission peak at around $400 \mathrm{~nm}$ as well as a broad defect related emission was observed in the EL spectra of the device. The turn-on voltage of the LED device is around $3 \mathrm{~V}$, favoring the low power consumption of the LED device. Also the NWs/PANi composite appears sufficiently robust to absorb large mechanical strain, which is also a fundamental issue in the practical use of organic/inorganic LEDs. Overall, this study demonstrates the possibility of using $\mathrm{ZnO}$ NWs/PANi heterojunction for blue light emission. We believe that the performance of such LED can be further improved through adjusting the thickness, carrier densities, and metal contact of the PANi layers.

\section{Acknowledgments}

The authors acknowledge the financial support of the NSFC (51072119), Innovation Program of Shanghai Municipal Education Commission (12ZZ139), and the Key Project of Chinese Ministry of Education (12057).

\section{References}

[1] S. Chu, G. Wang, W. Zhou et al., "Electrically pumped waveguide lasing from ZnO nanowires," Nature Nanotechnology, vol. 6, no. 8, pp. 506-510, 2011.

[2] E. Lai, W. Kim, and P. D. Yang, "Vertical nanowire array-based light emitting diodes," Nano Research, vol. 1, no. 2, pp. 123-128, 2008.
[3] X. W. Sun, J. Z. Huang, J. X. Wang, and Z. Xu, "A ZnO nanorod inorganic/organic heterostructure light-emitting diode emitting at 342 nm," Nano Letters, vol. 8, no. 4, pp. 1219-1223, 2008.

[4] R. Könenkamp, R. C. Word, and M. Godinez, "Ultraviolet electroluminescence from $\mathrm{ZnO}$ /polymer heterojunction lightemitting diodes," Nano Letters, vol. 5, no. 10, pp. 2005-2008, 2005.

[5] M. Law, L. E. Greene, J. C. Johnson, R. Saykally, and P. Yang, "Nanowire dye-sensitized solar cells," Nature Materials, vol. 4, no. 6, pp. 455-459, 2005.

[6] Ü. Ozgur, D. Hofstetter, and H. Morkoç, "ZnO devices and applications: a review of current status and future prospects," Proceedings of the IEEE, vol. 98, no. 7, pp. 1255-1268, 2010.

[7] S. Zaman, A. Zainelabdin, G. Amin, O. Nur, and M. Willander, "Influence of the polymer concentration on the electroluminescence of $\mathrm{ZnO}$ nanorod/polymer hybrid light emitting diodes," Journal of Applied Physics, vol. 112, no. 6, Article ID 064324, 6 pages, 2012.

[8] J. H. Jun, K. Cho, J. Yun, K. S. Suh, T. Kim, and S. Kim, "Enhancement of electrical characteristics of electrospun Polyaniline nanofibers by embedding the nanofibers with Ga-doped $\mathrm{ZnO}$ nanoparticles," Organic Electronics, vol. 9, no. 4, pp. 445451, 2008.

[9] J. Kim, S. Park, and N. F. Scherer, "Ultrafast dynamics of polarons in conductive polyaniline: comparison of primary and secondary doped forms," Journal of Physical Chemistry B, vol. 112, no. 49, pp. 15576-15587, 2008.

[10] D. P. Halliday, J. W. Gray, P. N. Adams, and A. P. Monkman, "Electrical and optical properties of a polymer semiconductor interface," Synthetic Metals, vol. 102, no. 1-3, pp. 877-878, 1999.

[11] S. W. Lee, D. O. Cho, G. Panin, and T. W. Kang, "Vertical ZnO nanowires/Si contact light emitting diode," Applied Physics Letters, vol. 98, Article ID 093110, 3 pages, 2011. 

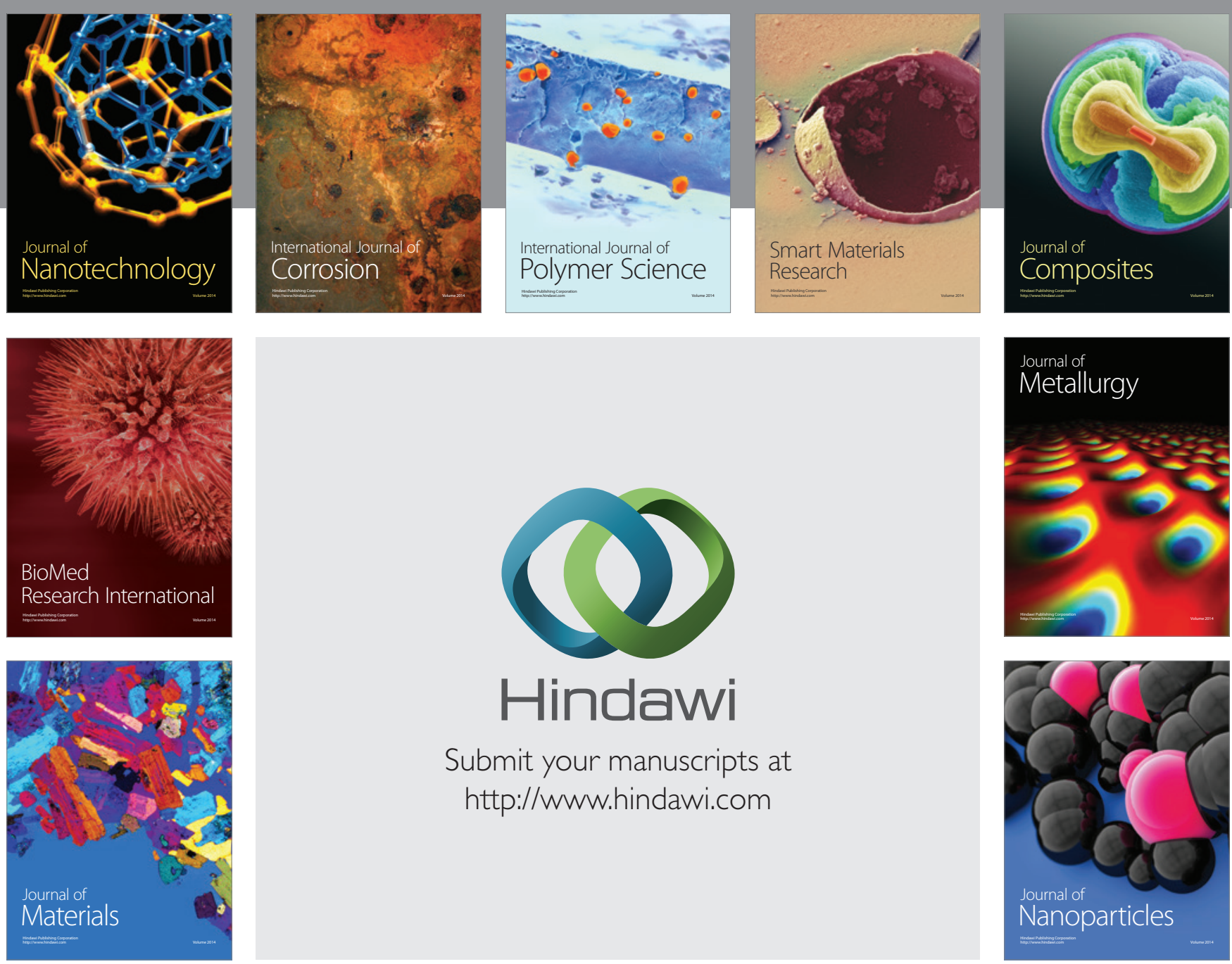

Submit your manuscripts at http://www.hindawi.com
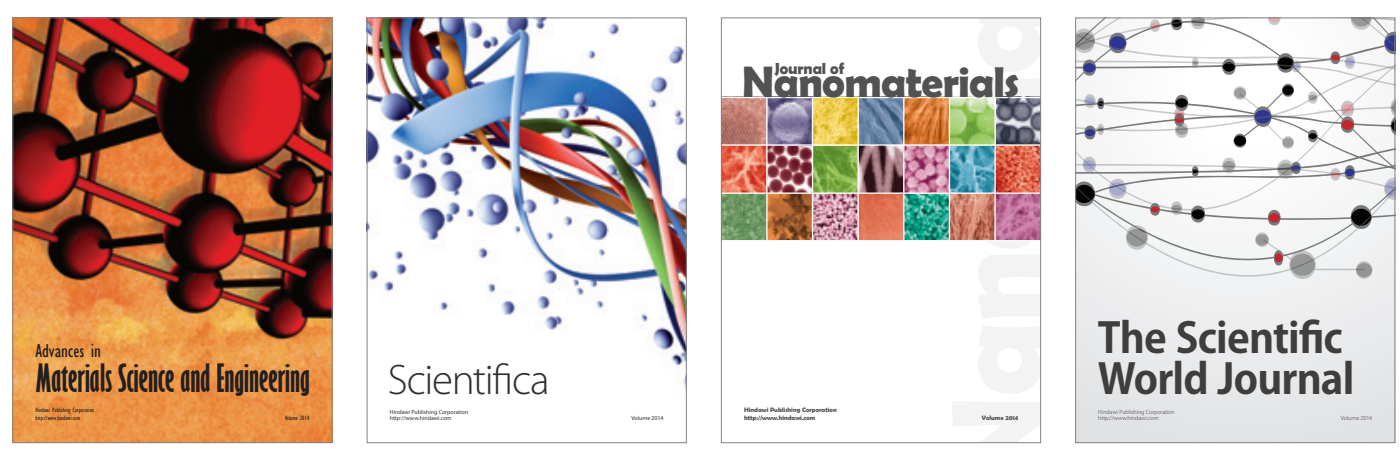

\section{The Scientific World Journal}
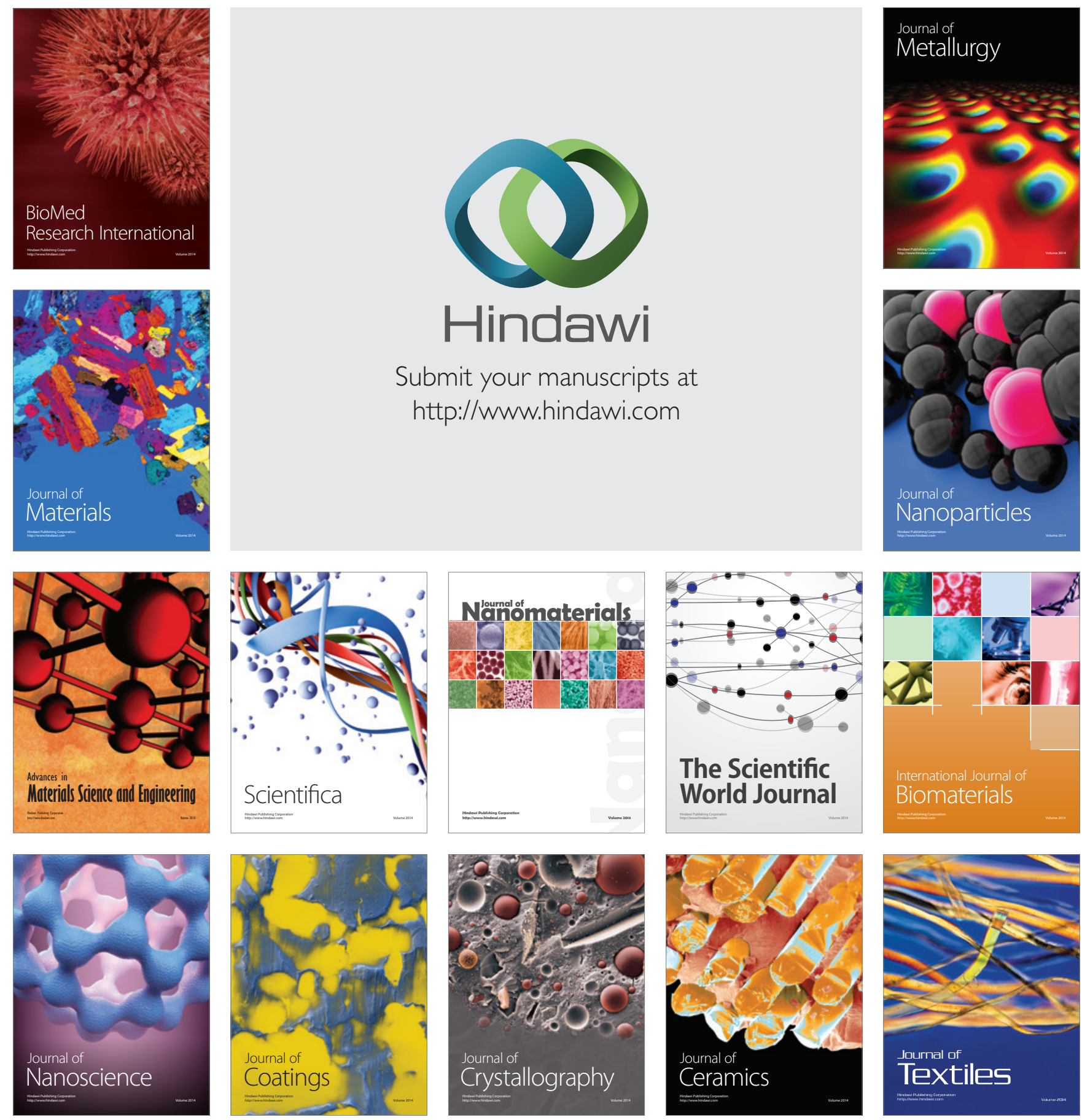